\title{
EXISTENCE OF POSITIVE PERIODIC SOLUTIONS FOR DELAYED PREDATOR-PREY PATCH SYSTEMS WITH STOCKING
}

HUI FANG AND ZHICHENG WANG

Received 28 July 2005; Revised 11 December 2005; Accepted 25 January 2006

A sufficient condition is derived for the existence of positive periodic solutions for a delayed predator-prey patch system with stocking. Some known results are improved.

Copyright (c) 2006 Hindawi Publishing Corporation. All rights reserved.

\section{Introduction}

Predator-prey systems have been studied extensively. See, for instance, $[1,6,8-10]$ and the references cited therein. Most of the previous papers focused on the predator-prey systems without stocking. Brauer and Soudack [2, 3] studied some predator-prey systems under constant rate stocking. To our knowledge, few papers have been published on the existence of positive periodic solutions for delayed predator-prey patch systems with periodic stocking.

In this paper, we investigate the following predator-prey system with stocking:

$$
\begin{gathered}
x_{1}^{\prime}(t)=x_{1}(t)\left(a_{1}(t)-b_{1}(t) x_{1}(t)-c(t) y(t)\right)+D_{1}(t)\left(x_{2}\left(t-\tau_{1}(t)\right)-x_{1}(t)\right)+S_{1}(t), \\
x_{2}^{\prime}(t)=x_{2}(t)\left(a_{2}(t)-b_{2}(t) x_{2}(t)\right)+D_{2}(t)\left(x_{1}\left(t-\tau_{2}(t)\right)-x_{2}(t)\right)+S_{2}(t), \\
y^{\prime}(t)=y(t)\left(-d(t)+p(t) x_{1}(t)-q(t) y(t)-\beta(t) \int_{-\tau}^{0} k(s) y(t+s) d s\right)+S_{3}(t),
\end{gathered}
$$

with the initial conditions

$$
\begin{array}{cc}
x_{1}(s)=\varphi_{1}(s) \geq 0, & s \in[-\sigma, 0], \varphi_{1}(0)>0, \\
x_{2}(s)=\varphi_{2}(s) \geq 0, & s \in[-\sigma, 0], \varphi_{2}(0)>0, \\
y(s)=\psi(s) \geq 0, & s \in[-\sigma, 0], \psi(0)>0,
\end{array}
$$

where $x_{1}$ and $y$ are the population densities of prey species $x$ and predator species $y$ in patch 1 , and $x_{2}$ is the density of species $x$ in patch 2. Predator species $y$ is confined to 
patch 1 , while the prey species $x$ can diffuse between two patches. $D_{i}(t)(i=1,2)$ are diffusion coefficients of species $x . S_{i}(t)(i=1,2,3)$ denote the stocking rates. $\varphi_{1}(s), \varphi_{2}(s)$, and $\psi(s)$ are continuous on $[-\sigma, 0], \sigma=\max \left\{\tau, \sup _{t \in \mathbb{R}} \tau_{1}(t), \sup _{t \in \mathbb{R}} \tau_{2}(t)\right\}$. The delay $\tau_{1}\left(\tau_{2}\right)$ represents the time that species $x$ migrates from patch 2 to patch 1 (patch 1 to patch 2 ).

When $S_{i}(t) \equiv 0(i=1,2,3), \tau_{i} \equiv 0(i=1,2)$, system (1.1) was considered by Zhang and Wang [15], Song and Chen [11], and Chen et al. [5].

The purpose of this paper is to derive a set of easily verifiable conditions for the existence of positive periodic solutions of system (1.1). The method in this paper is different from those of $[4,12-14]$.

\section{Existence of positive periodic solutions}

To show the existence of solutions to the considered problems, we will use an abstract theorem developed [7]. We first state this abstract theorem.

For a fixed $\sigma \geq 0$, let $\mathbb{C}:=\mathbb{C}\left([-\sigma, 0] ; \mathbb{R}^{n}\right)$. If $x \in \mathbb{C}\left([\gamma-\sigma, \gamma+\delta] ; \mathbb{R}^{n}\right)$ for some $\delta>0$ and $\gamma \in \mathbb{R}$, then $x_{t} \in \mathbb{C}$ for $t \in[\gamma, \gamma+\delta]$ is defined by $x_{t}(\theta)=x(t+\theta)$ for $\theta \in[-\sigma, 0]$. The supremum norm in $\mathbb{C}$ is denoted by $\|\cdot\|_{c}$, that is, $\|\phi\|_{c}=\max _{\theta \in[-\sigma, 0]}\|\phi(\theta)\|$ for $\phi \in \mathbb{C}$, where $\|\cdot\|$ denotes the norm in $\mathbb{R}^{n}$, and $\|u\|=\sum_{i=1}^{n}\left|u_{i}\right|$ for $u=\left(u_{1}, \ldots, u_{n}\right) \in \mathbb{R}^{n}$.

We consider the following functional differential equation:

$$
\frac{d x(t)}{d t}=f\left(t, x_{t}\right)
$$

where $f: \mathbb{R} \times \mathbb{C} \rightarrow \mathbb{R}^{n}$ is completely continuous, and there exists $T>0$ such that for every $(t, \varphi) \in \mathbb{R} \times \mathbb{C}$, we have $f(t+T, \varphi)=f(t, \varphi)$.

The following lemma is a simple consequence of [7, Theorem 4.7.1].

Lemma 2.1. Suppose that there exists a constant $M>0$ such that

(i) for any $\lambda \in(0,1)$ and any $T$-periodic solution $x$ of the system

$$
\frac{d x(t)}{d t}=\lambda f\left(t, x_{t}\right)
$$

$\|x(t)\|<M$ for $t \in \mathbb{R} ;$

(ii) $g(u):=(1 / T) \int_{0}^{T} f(s, \hat{u}) d s \neq 0$ for $u \in \partial B_{M}\left(\mathbb{R}^{n}\right)$, where $B_{M}\left(\mathbb{R}^{n}\right)=\left\{u \in \mathbb{R}^{n}:\|u\|<\right.$ $M\}$, and $\hat{u}$ denotes the constant mapping from $[-\sigma, 0]$ to $\mathbb{R}^{n}$ with the value $u \in \mathbb{R}^{n}$;

(iii) Brouwer degree $\operatorname{deg}\left(g, B_{M}\left(\mathbb{R}^{n}\right)\right) \neq 0$.

Then there exists at least one T-periodic solution of the system

$$
\frac{d x(t)}{d t}=f\left(t, x_{t}\right)
$$

that satisfies $\sup _{t \in \mathbb{R}}\|x(t)\|<M$.

In the following, we set

$$
\bar{g}=\frac{1}{T} \int_{0}^{T} g(t) d t, \quad g^{l}=\min _{t \in[0, T]}|g(t)|, \quad g^{u}=\max _{t \in[0, T]}|g(t)|,
$$

where $g$ is a continuous $T$-periodic function. 
In system (1.1), we always assume the following.

$\left(\mathrm{H}_{1}\right) a_{i}(t), b_{i}(t), D_{i}(t)(i=1,2), c(t), d(t), p(t), q(t)$, and $\beta(t)$ are positive continuous $T$-periodic functions. $S_{i}(t)(i=1,2,3), \tau_{i}(t)(i=1,2)$ are nonnegative continuous $T$-periodic functions. $\tau_{i}^{\prime}(t)<1(i=1,2), t \in \mathbb{R}$.

$\left(\mathrm{H}_{2}\right) k(s) \geq 0$ on $[-\tau, 0](0 \leq \tau<+\infty)$; and $k(s)$ is a piecewise continuous and normalized function such that $\int_{-\tau}^{0} k(s) d s=1$.

Set

$$
\begin{gathered}
K=\frac{\bar{q}+\bar{\beta}}{\bar{p}}, \\
K^{*}=\left(\frac{a_{1} M_{0}-D_{1} M_{0}+S_{1}}{b_{1} M_{0}}\right)^{l}, \quad K_{i}^{*}=\left(\frac{a_{i} M_{0}+S_{i}}{b_{i} M_{0}}\right)^{l}, \quad i=1,2, \\
M_{0}=\max \left\{\left(\frac{a_{1}+\sqrt{a_{1}^{2}+4 b_{1} S_{1}}}{2 b_{1}}\right)^{u},\left(\frac{a_{2}+\sqrt{a_{2}^{2}+4 b_{2} S_{2}}}{2 b_{2}}\right)^{u}\right\}, \\
m_{0}=\min \left\{\frac{\left(a_{1} / c\right)^{l}-\sqrt{\left(S_{3} / q\right)^{u}}}{b_{1}^{u} / c^{l}+(p / q)^{u}} \exp \left[-2 T\left(\bar{D}_{1}+\bar{b}_{1} M_{0}+\bar{c} \widetilde{M}_{0}\right)\right],\left(\frac{a_{2}+\sqrt{a_{2}^{2}+4 b_{2} S_{2}}}{2 b_{2}}\right)^{l}\right\}, \\
\tilde{m}_{0}=\min \left\{\frac{K_{1}^{*}-\bar{d} / \bar{p}}{K+\left(c / b_{1}\right)^{u}}, \frac{K_{2}^{*}-\bar{d} / \bar{p}}{K}, \frac{K^{*}-\bar{d} / \bar{p}}{K+\left(c / b_{1}\right)^{u}}\right\} \exp \left[-2 T\left(\bar{d}+\bar{q} \widetilde{M}_{0}+\bar{\beta} \widetilde{M}_{0}\right)\right] .
\end{gathered}
$$

Theorem 2.2. In addition to $\left(\mathrm{H}_{1}\right),\left(\mathrm{H}_{2}\right)$, assume further that system (1.1) satisfies one of the following assumptions:

$\left(\mathrm{H}_{3}\right)\left(a_{1} / c\right)^{l}>\sqrt{\left(S_{3} / q\right)^{u}}, K_{i}^{*}>\bar{d} / \bar{p}(i=1,2)$;

$\left(\mathrm{H}_{4}\right)\left(a_{1} / c\right)^{l}>\sqrt{\left(S_{3} / q\right)^{u}}, K^{*}>\bar{d} / \bar{p}$.

Then system (1.1) has at least one positive T-periodic solution, say $\left(x_{1}^{*}(t), x_{2}^{*}(t), y^{*}(t)\right)^{T}$ such that

$$
m_{0} \leq x_{i}^{*}(t) \leq M_{0} \quad(i=1,2), \quad \tilde{m}_{0} \leq y^{*}(t) \leq \widetilde{M}_{0}, \quad t \geq 0 .
$$

Proof. Consider the following system:

$$
\begin{gathered}
u_{1}^{\prime}(t)=a_{1}(t)-D_{1}(t)-b_{1}(t) e^{u_{1}(t)}-c(t) e^{u_{3}(t)}+D_{1}(t) e^{u_{2}\left(t-\tau_{1}(t)\right)-u_{1}(t)}+\frac{S_{1}(t)}{e^{u_{1}(t)}}, \\
u_{2}^{\prime}(t)=a_{2}(t)-D_{2}(t)-b_{2}(t) e^{u_{2}(t)}+D_{2}(t) e^{u_{1}\left(t-\tau_{2}(t)\right)-u_{2}(t)}+\frac{S_{2}(t)}{e^{u_{2}(t)}} \\
u_{3}^{\prime}(t)=-d(t)+p(t) e^{u_{1}(t)}-q(t) e^{u_{3}(t)}-\beta(t) \int_{-\tau}^{0} k(s) e^{u_{3}(t+s)} d s+\frac{S_{3}(t)}{e^{u_{3}(t)}}
\end{gathered}
$$


4 Periodic solutions for predator-prey patch systems

where $a_{i}(t), b_{i}(t), D_{i}(t)(i=1,2), S_{i}(t)(i=1,2,3), c(t), d(t), p(t), q(t)$, and $\beta(t)$ are the same as those in assumption $\left(\mathrm{H}_{1}\right)$, and $\tau, \tau_{i}(i=1,2)$ and $k(s)$ are the same as those in assumption $\left(\mathrm{H}_{2}\right)$. We first show that system $(2.7)$ has one $T$-periodic solution.

Let $\mathbb{C}:=\mathbb{C}\left([-\sigma, 0] ; \mathbb{R}^{3}\right)$. We define the following map:

$$
\begin{gathered}
f: \mathbb{R} \times \mathbb{C} \longrightarrow \mathbb{R}^{3}, \quad f(t, \varphi)=\left(f_{1}(t, \varphi), f_{2}(t, \varphi), f_{3}(t, \varphi)\right), \quad \varphi=\left(\varphi_{1}, \varphi_{2}, \varphi_{3}\right) \in \mathbb{C}, \\
f_{1}(t, \varphi)=a_{1}(t)-D_{1}(t)-b_{1}(t) e^{\varphi_{1}(0)}-c(t) e^{\varphi_{3}(0)}+D_{1}(t) e^{\varphi_{2}\left(-\tau_{1}(t)\right)-\varphi_{1}(0)}+\frac{S_{1}(t)}{e^{\varphi_{1}(0)}}, \\
f_{2}(t, \varphi)=a_{2}(t)-D_{2}(t)-b_{2}(t) e^{\varphi_{2}(0)}+D_{2}(t) e^{\varphi_{1}\left(-\tau_{2}(t)\right)-\varphi_{2}(0)}+\frac{S_{2}(t)}{e^{\varphi_{2}(0)}}, \\
f_{3}(t, \varphi)=-d(t)+p(t) e^{\varphi_{1}(0)}-q(t) e^{\varphi_{3}(0)}-\beta(t) \int_{-\tau}^{0} k(s) e^{\varphi_{3}(s)} d s+\frac{S_{3}(t)}{e^{\varphi_{3}(0)}} .
\end{gathered}
$$

Clearly, $f: \mathbb{R} \times \mathbb{C} \rightarrow \mathbb{R}^{3}$ is completely continuous. Now, the system (2.7) becomes

$$
\frac{d u(t)}{d t}=f\left(t, u_{t}\right)
$$

Corresponding to

$$
\frac{d u(t)}{d t}=\lambda f\left(t, u_{t}\right), \quad \lambda \in(0,1)
$$

we have

$$
\begin{aligned}
& u_{1}^{\prime}(t)=\lambda\left[a_{1}(t)-D_{1}(t)-b_{1}(t) e^{u_{1}(t)}-c(t) e^{u_{3}(t)}+D_{1}(t) e^{u_{2}\left(t-\tau_{1}(t)\right)-u_{1}(t)}+\frac{S_{1}(t)}{e^{u_{1}(t)}}\right], \\
& u_{2}^{\prime}(t)=\lambda\left[a_{2}(t)-D_{2}(t)-b_{2}(t) e^{u_{2}(t)}+D_{2}(t) e^{u_{1}\left(t-\tau_{2}(t)\right)-u_{2}(t)}+\frac{S_{2}(t)}{e^{u_{2}(t)}}\right], \\
& u_{3}^{\prime}(t)=\lambda\left[-d(t)+p(t) e^{u_{1}(t)}-q(t) e^{u_{3}(t)}-\beta(t) \int_{-\tau}^{0} k(s) e^{u_{3}(t+s)} d s+\frac{S_{3}(t)}{e^{u_{3}(t)}}\right] .
\end{aligned}
$$

Suppose that $\left(u_{1}(t), u_{2}(t), u_{3}(t)\right)^{T}$ is a $T$-periodic solution of system (2.11) for some $\lambda \in$ $(0,1)$. Choose $t_{i}^{M}, t_{i}^{m} \in[0, T], i=1,2,3$, such that

$$
u_{i}\left(t_{i}^{M}\right)=\max _{t \in[0, T]} u_{i}(t), \quad u_{i}\left(t_{i}^{m}\right)=\min _{t \in[0, T]} u_{i}(t), \quad i=1,2,3
$$

Then, it is clear that

$$
u_{i}^{\prime}\left(t_{i}^{M}\right)=0, \quad u_{i}^{\prime}\left(t_{i}^{m}\right)=0, \quad i=1,2,3 .
$$


From this and system (2.11), we obtain that

$$
\begin{gathered}
a_{1}\left(t_{1}^{M}\right)-D_{1}\left(t_{1}^{M}\right)-b_{1}\left(t_{1}^{M}\right) e^{u_{1}\left(t_{1}^{M}\right)}-c\left(t_{1}^{M}\right) e^{u_{3}\left(t_{1}^{M}\right)}+D_{1}\left(t_{1}^{M}\right) e^{u_{2}\left(t_{1}^{M}-\tau_{1}\left(t_{1}^{M}\right)\right)-u_{1}\left(t_{1}^{M}\right)}+\frac{S_{1}\left(t_{1}^{M}\right)}{e^{u_{1}\left(t_{1}^{M}\right)}}=0, \\
a_{2}\left(t_{2}^{M}\right)-D_{2}\left(t_{2}^{M}\right)-b_{2}\left(t_{2}^{M}\right) e^{u_{2}\left(t_{2}^{M}\right)}+D_{2}\left(t_{2}^{M}\right) e^{u_{1}\left(t_{2}^{M}-\tau_{2}\left(t_{2}^{M}\right)\right)-u_{2}\left(t_{2}^{M}\right)}+\frac{S_{2}\left(t_{2}^{M}\right)}{e^{u_{2}\left(t_{2}^{M}\right)}}=0, \\
-d\left(t_{3}^{M}\right)+p\left(t_{3}^{M}\right) e^{u_{1}\left(t_{3}^{M}\right)}-q\left(t_{3}^{M}\right) e^{u_{3}\left(t_{3}^{M}\right)}-\beta\left(t_{3}^{M}\right) \int_{-\tau}^{0} k(s) e^{u_{3}\left(t_{3}^{M}+s\right)} d s+\frac{S_{3}\left(t_{3}^{M}\right)}{e^{u_{3}\left(t_{3}^{M}\right)}}=0, \\
a_{1}\left(t_{1}^{m}\right)-D_{1}\left(t_{1}^{m}\right)-b_{1}\left(t_{1}^{m}\right) e^{u_{1}\left(t_{1}^{m}\right)}-c\left(t_{1}^{m}\right) e^{u_{3}\left(t_{1}^{m}\right)}+D_{1}\left(t_{1}^{m}\right) e^{u_{2}\left(t_{1}^{m}-\tau_{1}\left(t_{1}^{m}\right)\right)-u_{1}\left(t_{1}^{m}\right)}+\frac{S_{1}\left(t_{1}^{m}\right)}{e^{u_{1}\left(t_{1}^{m}\right)}}=0, \\
a_{2}\left(t_{2}^{m}\right)-D_{2}\left(t_{2}^{m}\right)-b_{2}\left(t_{2}^{m}\right) e^{u_{2}\left(t_{2}^{m}\right)}+D_{2}\left(t_{2}^{m}\right) e^{u_{1}\left(t_{2}^{m}-\tau_{2}\left(t_{2}^{m}\right)\right)-u_{2}\left(t_{2}^{m}\right)}+\frac{S_{2}\left(t_{2}^{m}\right)}{e^{u_{2}\left(t_{2}^{m}\right)}}=0 .
\end{gathered}
$$

Next we make the following claims.

Claim 1. For $u_{i}\left(t_{i}^{M}\right)(i=1,2)$, one of the following cases holds:

$$
\begin{aligned}
& u_{2}\left(t_{2}^{M}\right) \leq u_{1}\left(t_{1}^{M}\right) \leq M_{1}^{*} \leq M_{1}, \\
& u_{1}\left(t_{1}^{M}\right)<u_{2}\left(t_{2}^{M}\right) \leq M_{2}^{*} \leq M_{1},
\end{aligned}
$$

where $M_{1}:=\max \left\{M_{1}^{*}, M_{2}^{*}\right\}, M_{j}^{*}:=\ln \left(\left(a_{j}+\sqrt{a_{j}^{2}+4 b_{j} S_{j}}\right) / 2 b_{j}\right)^{u}, j=1,2$.

There are two cases to consider.

Case 1. Assume that $u_{1}\left(t_{1}^{M}\right) \geq u_{2}\left(t_{2}^{M}\right)$; then $u_{1}\left(t_{1}^{M}\right) \geq u_{2}\left(t_{1}^{M}-\tau_{1}\left(t_{1}^{M}\right)\right)$.

From this and (2.14), we have

$$
b_{1}\left(t_{1}^{M}\right) e^{u_{1}\left(t_{1}^{M}\right)} \leq a_{1}\left(t_{1}^{M}\right)+\frac{S_{1}\left(t_{1}^{M}\right)}{e^{u_{1}\left(t_{1}^{M}\right)}}
$$

That is,

$$
b_{1}\left(t_{1}^{M}\right) e^{2 u_{1}\left(t_{1}^{M}\right)}-a_{1}\left(t_{1}^{M}\right) e^{u_{1}\left(t_{1}^{M}\right)}-S_{1}\left(t_{1}^{M}\right) \leq 0
$$

Therefore,

$$
e^{u_{1}\left(t_{1}^{M}\right)} \leq \frac{a_{1}\left(t_{1}^{M}\right)+\sqrt{a_{1}^{2}\left(t_{1}^{M}\right)+4 b_{1}\left(t_{1}^{M}\right) S_{1}\left(t_{1}^{M}\right)}}{2 b_{1}\left(t_{1}^{M}\right)} \leq\left(\frac{a_{1}+\sqrt{a_{1}^{2}+4 b_{1} S_{1}}}{2 b_{1}}\right)^{u} .
$$

Hence,

$$
u_{2}\left(t_{2}^{M}\right) \leq u_{1}\left(t_{1}^{M}\right) \leq \ln \left(\frac{a_{1}+\sqrt{a_{1}^{2}+4 b_{1} S_{1}}}{2 b_{1}}\right)^{u} .
$$


6 Periodic solutions for predator-prey patch systems

Case 2. Assume that $u_{1}\left(t_{1}^{M}\right)<u_{2}\left(t_{2}^{M}\right)$; then $u_{1}\left(t_{2}^{M}-\tau_{2}\left(t_{2}^{M}\right)\right)<u_{2}\left(t_{2}^{M}\right)$.

From this and (2.15), we have

$$
b_{2}\left(t_{2}^{M}\right) e^{u_{2}\left(t_{2}^{M}\right)} \leq a_{2}\left(t_{2}^{M}\right)+\frac{S_{2}\left(t_{2}^{M}\right)}{e^{u_{2}\left(t_{2}^{M}\right)}} .
$$

By a similar argument to Case 1 , we have

$$
u_{1}\left(t_{1}^{M}\right)<u_{2}\left(t_{2}^{M}\right) \leq \ln \left(\frac{a_{2}+\sqrt{a_{2}^{2}+4 b_{2} S_{2}}}{2 b_{2}}\right)^{u} .
$$

It follows from (2.24) and (2.26) that Claim 1 holds.

Claim 2.

$$
u_{3}\left(t_{3}^{M}\right) \leq \ln \left(\frac{p M_{0}+\sqrt{p^{2} M_{0}^{2}+4 q S_{3}}}{2 q}\right)^{u}:=M_{2},
$$

where $M_{0}=e^{M_{1}}$.

By (2.16), we have

$$
q\left(t_{3}^{M}\right) e^{u_{3}\left(t_{3}^{M}\right)} \leq p\left(t_{3}^{M}\right) e^{u_{1}\left(t_{3}^{M}\right)}+\frac{S_{3}\left(t_{3}^{M}\right)}{e^{u_{3}\left(t_{3}^{M}\right)}} \leq p\left(t_{3}^{M}\right) e^{u_{1}\left(t_{1}^{M}\right)}+\frac{S_{3}\left(t_{3}^{M}\right)}{e^{u_{3}\left(t_{3}^{M}\right)}} .
$$

That is,

$$
q\left(t_{3}^{M}\right) e^{2 u_{3}\left(t_{3}^{M}\right)}-p\left(t_{3}^{M}\right) e^{u_{1}\left(t_{1}^{M}\right)} e^{u_{3}\left(t_{3}^{M}\right)}-S_{3}\left(t_{3}^{M}\right) \leq 0 .
$$

Therefore,

$$
e^{u_{3}\left(t_{3}^{M}\right)} \leq \frac{p\left(t_{3}^{M}\right) e^{u_{1}\left(t_{1}^{M}\right)}+\sqrt{p^{2}\left(t_{3}^{M}\right) e^{2 u_{1}\left(t_{1}^{M}\right)}+4 q\left(t_{3}^{M}\right) S_{3}\left(t_{3}^{M}\right)}}{2 q\left(t_{3}^{M}\right)},
$$

which implies that Claim 2 holds.

Claim 3. For $u_{i}\left(t_{i}^{m}\right)(i=1,2)$, one of the following cases holds:

$$
\begin{gathered}
m_{1} \leq m_{1}^{*}-2 T\left(\bar{D}_{1}+\bar{b}_{1} M_{0}+\bar{c} \widetilde{M}_{0}\right) \leq u_{1}\left(t_{1}^{m}\right) \leq u_{2}\left(t_{2}^{m}\right) \\
m_{1} \leq m_{2}^{*} \leq u_{2}\left(t_{2}^{m}\right)<u_{1}\left(t_{1}^{m}\right)
\end{gathered}
$$

where

$$
\begin{gathered}
m_{1}:=\min \left\{m_{1}^{*}-2 T\left(\bar{D}_{1}+\bar{b}_{1} M_{0}+\bar{c} \widetilde{M}_{0}\right), m_{2}^{*}\right\} \\
m_{1}^{*}:=\ln \frac{\left(a_{1} / c\right)^{l}-\sqrt{\left(S_{3} / q\right)^{u}}}{b_{1}^{u} / c^{l}+(p / q)^{u}} \\
m_{2}^{*}:=\ln \left(\frac{a_{2}+\sqrt{a_{2}^{2}+4 b_{2} S_{2}}}{2 b_{2}}\right)^{l}
\end{gathered}
$$

There are two cases to consider. 
Case 1. Assume that $u_{1}\left(t_{1}^{m}\right) \leq u_{2}\left(t_{2}^{m}\right)$; then $u_{1}\left(t_{1}^{m}\right) \leq u_{2}\left(t_{1}^{m}-\tau_{1}\left(t_{1}^{m}\right)\right)$.

From this and (2.17), we have

$$
a_{1}\left(t_{1}^{m}\right) \leq b_{1}\left(t_{1}^{m}\right) e^{u_{1}\left(t_{1}^{m}\right)}+c\left(t_{1}^{m}\right) e^{u_{3}\left(t_{1}^{m}\right)} \leq b_{1}\left(t_{1}^{m}\right) e^{u_{1}\left(t_{1}^{M}\right)}+c\left(t_{1}^{m}\right) e^{u_{3}\left(t_{3}^{M}\right)} .
$$

From (2.30), by using the inequality

$$
(a+b)^{1 / 2}<a^{1 / 2}+b^{1 / 2}, \quad a>0, b>0,
$$

we have

$$
e^{u_{3}\left(t_{3}^{M}\right)}<\frac{p\left(t_{3}^{M}\right) e^{u_{1}\left(t_{1}^{M}\right)}+\sqrt{q\left(t_{3}^{M}\right) S_{3}\left(t_{3}^{M}\right)}}{q\left(t_{3}^{M}\right)} .
$$

From this and (2.33), we have

$$
a_{1}\left(t_{1}^{m}\right) \leq\left[b_{1}\left(t_{1}^{m}\right)+\frac{c\left(t_{1}^{m}\right) p\left(t_{3}^{M}\right)}{q\left(t_{3}^{M}\right)}\right] e^{u_{1}\left(t_{1}^{M}\right)}+c\left(t_{1}^{m}\right) \sqrt{\frac{S_{3}\left(t_{3}^{M}\right)}{q\left(t_{3}^{M}\right)}}
$$

which implies

$$
\left(\frac{a_{1}}{c}\right)^{l} \leq\left[\frac{b_{1}^{u}}{c^{l}}+\left(\frac{p}{q}\right)^{u}\right] e^{u_{1}\left(t_{1}^{M}\right)}+\sqrt{\left(\frac{S_{3}}{q}\right)^{u}}
$$

That is,

$$
u_{1}\left(t_{1}^{M}\right) \geq \ln \frac{\left(a_{1} / c\right)^{l}-\sqrt{\left(S_{3} / q\right)^{u}}}{b_{1}^{u} / c^{l}+(p / q)^{u}}:=m_{1}^{*}
$$

From the first equation of system (2.11), we obtain that

$$
\begin{gathered}
\int_{0}^{T} a_{1}(t) d t+\int_{0}^{T} D_{1}(t) e^{u_{2}\left(t-\tau_{1}(t)\right)-u_{1}(t)} d t+\int_{0}^{T} \frac{S_{1}(t)}{e^{u_{1}(t)}} d t \\
=\int_{0}^{T} D_{1}(t) d t+\int_{0}^{T} b_{1}(t) e^{u_{1}(t)} d t+\int_{0}^{T} c(t) e^{u_{3}(t)} d t \\
\int_{0}^{T}\left|u_{1}^{\prime}(t)\right| d t<\int_{0}^{T} a_{1}(t) d t+\int_{0}^{T} D_{1}(t) e^{u_{2}\left(t-\tau_{1}(t)\right)-u_{1}(t)} d t+\int_{0}^{T} \frac{S_{1}(t)}{e^{u_{1}(t)}} d t \\
+\int_{0}^{T} D_{1}(t) d t+\int_{0}^{T} b_{1}(t) e^{u_{1}(t)} d t+\int_{0}^{T} c(t) e^{u_{3}(t)} d t .
\end{gathered}
$$


8 Periodic solutions for predator-prey patch systems

It follows that

$$
\begin{aligned}
\int_{0}^{T}\left|u_{1}^{\prime}(t)\right| d t & <2\left[\int_{0}^{T} D_{1}(t) d t+\int_{0}^{T} b_{1}(t) e^{u_{1}(t)} d t+\int_{0}^{T} c(t) e^{u_{3}(t)} d t\right] \\
& \leq 2\left[\int_{0}^{T} D_{1}(t) d t+e^{M_{1}} \int_{0}^{T} b_{1}(t) d t+e^{M_{2}} \int_{0}^{T} c(t) d t\right] \\
& =2 T\left(\bar{D}_{1}+\bar{b}_{1} M_{0}+\bar{c} \widetilde{M}_{0}\right) .
\end{aligned}
$$

From (2.38) and (2.40), we have

$$
u_{1}\left(t_{1}^{m}\right) \geq u_{1}\left(t_{1}^{M}\right)-\int_{0}^{T}\left|u_{1}^{\prime}(t)\right| d t \geq m_{1}^{*}-2 T\left(\bar{D}_{1}+\bar{b}_{1} M_{0}+\bar{c} \widetilde{M}_{0}\right) .
$$

Case 2. Assume that $u_{1}\left(t_{1}^{m}\right)>u_{2}\left(t_{2}^{m}\right)$; then $u_{1}\left(t_{2}^{m}-\tau_{2}\left(t_{2}^{m}\right)\right)>u_{2}\left(t_{2}^{m}\right)$.

From this and (2.18), we have

$$
b_{2}\left(t_{2}^{m}\right) e^{u_{2}\left(t_{2}^{m}\right)} \geq a_{2}\left(t_{2}^{m}\right)+\frac{S_{2}\left(t_{2}^{m}\right)}{e^{u_{2}\left(t_{2}^{m}\right)}}
$$

which implies

$$
e^{u_{2}\left(t_{2}^{m}\right)} \geq \frac{a_{2}\left(t_{2}^{m}\right)+\sqrt{a_{2}^{2}\left(t_{2}^{m}\right)+4 b_{2}\left(t_{2}^{m}\right) S_{2}\left(t_{2}^{m}\right)}}{2 b_{2}\left(t_{2}^{m}\right)} .
$$

That is,

$$
u_{2}\left(t_{2}^{m}\right) \geq \ln \left(\frac{a_{2}+\sqrt{a_{2}^{2}+4 b_{2} S_{2}}}{2 b_{2}}\right)^{l}:=m_{2}^{*} .
$$

It follows from (2.41) and (2.44) that Claim 3 holds.

Claim 4.

$$
u_{3}\left(t_{3}^{m}\right) \geq \min \left\{m_{3}^{*}, m_{4}^{*}, m_{5}^{*}\right\}-2 T\left(\bar{d}+\bar{q} \widetilde{M}_{0}+\bar{\beta} \widetilde{M}_{0}\right):=m_{2},
$$

where

$$
m_{3}^{*}=\ln \frac{K_{1}^{*}-\bar{d} / \bar{p}}{K+\left(c / b_{1}\right)^{u}}, \quad m_{4}^{*}=\ln \frac{K_{2}^{*}-\bar{d} / \bar{p}}{K}, \quad m_{5}^{*}=\ln \frac{K^{*}-\bar{d} / \bar{p}}{K+\left(c / b_{1}\right)^{u}} .
$$

From the third equation of (2.11), we obtain

$$
\begin{gathered}
\int_{0}^{T} p(t) e^{u_{1}(t)} d t+\int_{0}^{T} \frac{S_{3}(t)}{e^{u_{3}(t)}} d t=\int_{0}^{T} d(t) d t+\int_{0}^{T} q(t) e^{u_{3}(t)} d t+\int_{0}^{T} \beta(t) \int_{-\tau}^{0} k(s) e^{u_{3}(t+s)} d s d t \\
\int_{0}^{T}\left|u_{3}^{\prime}(t)\right| d t<\int_{0}^{T} p(t) e^{u_{1}(t)} d t+\int_{0}^{T} \frac{S_{3}(t)}{e^{u_{3}(t)}} d t+\int_{0}^{T} d(t) d t \\
+\int_{0}^{T} q(t) e^{u_{3}(t)} d t+\int_{0}^{T} \beta(t) \int_{-\tau}^{0} k(s) e^{u_{3}(t+s)} d s d t .
\end{gathered}
$$


It follows that

$$
\begin{aligned}
& \int_{0}^{T}\left|u_{3}^{\prime}(t)\right| d t<2\left[\int_{0}^{T} d(t) d t+\int_{0}^{T} q(t) e^{u_{3}(t)} d t+\int_{0}^{T} \beta(t) \int_{-\tau}^{0} k(s) e^{u_{3}(t+s)} d s d t\right] \\
& \leq 2\left[\int_{0}^{T} d(t) d t+e^{M_{2}} \int_{0}^{T} q(t) d t+e^{M_{2}} \int_{0}^{T} \beta(t) d t\right] \\
&=2 T\left(\bar{d}+\bar{q} \widetilde{M}_{0}+\bar{\beta} \widetilde{M}_{0}\right), \\
& {[\bar{q}+\bar{\beta}] e^{u_{3}\left(t_{3}^{M}\right)} \geq \bar{p} e^{u_{1}\left(t_{1}^{m}\right)}-\bar{d} . }
\end{aligned}
$$

There are two cases to consider.

Case 1. Assume that the assumption $\left(\mathrm{H}_{3}\right)$ holds.

If $u_{1}\left(t_{1}^{m}\right) \leq u_{2}\left(t_{2}^{m}\right)$, by (2.17), we have

$$
\begin{aligned}
e^{u_{1}\left(t_{1}^{m}\right)} & \geq \frac{a_{1}\left(t_{1}^{m}\right)-c\left(t_{1}^{m}\right) e^{u_{3}\left(t_{1}^{m}\right)}}{b_{1}\left(t_{1}^{m}\right)}+\frac{S_{1}\left(t_{1}^{m}\right)}{b_{1}\left(t_{1}^{m}\right) e^{u_{1}\left(t_{1}^{m}\right)}} \\
& \geq \frac{a_{1}\left(t_{1}^{m}\right)-c\left(t_{1}^{m}\right) e^{u_{3}\left(t_{3}^{M}\right)}}{b_{1}\left(t_{1}^{m}\right)}+\frac{S_{1}\left(t_{1}^{m}\right)}{b_{1}\left(t_{1}^{m}\right) e^{M_{1}}} .
\end{aligned}
$$

Substituting this into (2.49) gives

$$
[\bar{q}+\bar{\beta}] e^{u_{3}\left(t_{3}^{M}\right)} \geq \frac{\bar{p} a_{1}\left(t_{1}^{m}\right)}{b_{1}\left(t_{1}^{m}\right)}-\frac{\bar{p} c\left(t_{1}^{m}\right) e^{u_{3}\left(t_{3}^{M}\right)}}{b_{1}\left(t_{1}^{m}\right)}+\frac{\bar{p} S_{1}\left(t_{1}^{m}\right)}{b_{1}\left(t_{1}^{m}\right) e^{M_{1}}}-\bar{d},
$$

which implies

$$
\left[\frac{\bar{q}}{\bar{p}}+\frac{\bar{\beta}}{\bar{p}}+\frac{c\left(t_{1}^{m}\right)}{b_{1}\left(t_{1}^{m}\right)}\right] e^{u_{3}\left(t_{3}^{M}\right)} \geq \frac{a_{1}\left(t_{1}^{m}\right)}{b_{1}\left(t_{1}^{m}\right)}+\frac{S_{1}\left(t_{1}^{m}\right)}{b_{1}\left(t_{1}^{m}\right) e^{M_{1}}}-\frac{\bar{d}}{\bar{p}}
$$

Therefore,

$$
\left[K+\left(\frac{c}{b_{1}}\right)^{u}\right] e^{u_{3}\left(t_{3}^{M}\right)} \geq K_{1}^{*}-\frac{\bar{d}}{\bar{p}}
$$

That is,

$$
u_{3}\left(t_{3}^{M}\right) \geq \ln \frac{K_{1}^{*}-\bar{d} / \bar{p}}{K+\left(c / b_{1}\right)^{u}}:=m_{3}^{*} .
$$

It follows from (2.48) and (2.54)that

$$
u_{3}\left(t_{3}^{m}\right) \geq u_{3}\left(t_{3}^{M}\right)-\int_{0}^{T}\left|u_{3}^{\prime}(t)\right| d t \geq m_{3}^{*}-2 T\left(\bar{d}+\bar{q} \widetilde{M}_{0}+\bar{\beta} \widetilde{M}_{0}\right) .
$$

If $u_{1}\left(t_{1}^{m}\right)>u_{2}\left(t_{2}^{m}\right)$, by (2.42), (2.49), and (2.19), we have

$$
[\bar{q}+\bar{\beta}] e^{u_{3}\left(t_{3}^{M}\right)} \geq \bar{p} e^{u_{2}\left(t_{2}^{m}\right)}-\bar{d} \geq \frac{\bar{p}\left[a_{2}\left(t_{2}^{m}\right)+S_{2}\left(t_{2}^{m}\right) e^{-M_{1}}\right]}{b_{2}\left(t_{2}^{m}\right)}-\bar{d},
$$


which implies

$$
\left[\frac{\bar{q}}{\bar{p}}+\frac{\bar{\beta}}{\bar{p}}\right] e^{u_{3}\left(t_{3}^{M}\right)} \geq \frac{a_{2}\left(t_{2}^{m}\right)+S_{2}\left(t_{2}^{m}\right) e^{-M_{1}}}{b_{2}\left(t_{2}^{m}\right)}-\frac{\bar{d}}{\bar{p}} .
$$

Therefore,

$$
K e^{u_{3}\left(t_{3}^{M}\right)} \geq K_{2}^{*}-\frac{\bar{d}}{\bar{p}}
$$

That is,

$$
u_{3}\left(t_{3}^{M}\right) \geq \ln \frac{K_{2}^{*}-\bar{d} / \bar{p}}{K}:=m_{4}^{*} .
$$

From (2.48) and (2.59), we have

$$
u_{3}\left(t_{3}^{m}\right) \geq u_{3}\left(t_{3}^{M}\right)-\int_{0}^{T}\left|u_{3}^{\prime}(t)\right| d t \geq m_{4}^{*}-2 T\left(\bar{d}+\bar{q} \widetilde{M}_{0}+\bar{\beta} \widetilde{M}_{0}\right) .
$$

Case 2. Assume that the assumption $\left(\mathrm{H}_{4}\right)$ holds.

From (2.17), we have

$$
\begin{aligned}
b_{1}\left(t_{1}^{m}\right) e^{u_{1}\left(t_{1}^{m}\right)} & \geq a_{1}\left(t_{1}^{m}\right)-D_{1}\left(t_{1}^{m}\right)-c\left(t_{1}^{m}\right) e^{u_{3}\left(t_{1}^{m}\right)}+\frac{S_{1}\left(t_{1}^{m}\right)}{e^{u_{1}\left(t_{1}^{m}\right)}} \\
& \geq a_{1}\left(t_{1}^{m}\right)-D_{1}\left(t_{1}^{m}\right)-c\left(t_{1}^{m}\right) e^{u_{3}\left(t_{3}^{M}\right)}+\frac{S_{1}\left(t_{1}^{m}\right)}{e^{M_{1}}} .
\end{aligned}
$$

Therefore,

$$
e^{u_{1}\left(t_{1}^{m}\right)} \geq \frac{a_{1}\left(t_{1}^{m}\right)-D_{1}\left(t_{1}^{m}\right)-c\left(t_{1}^{m}\right) e^{u_{3}\left(t_{3}^{M}\right)}+S_{1}\left(t_{1}^{m}\right) e^{-M_{1}}}{b_{1}\left(t_{1}^{m}\right)} .
$$

Substituting this into (2.49) gives

$$
[\bar{q}+\bar{\beta}] e^{u_{3}\left(t_{3}^{M}\right)} \geq \frac{\bar{p}\left[a_{1}\left(t_{1}^{m}\right)-D_{1}\left(t_{1}^{m}\right)\right]}{b_{1}\left(t_{1}^{m}\right)}-\frac{\bar{p} c\left(t_{1}^{m}\right) e^{u_{3}\left(t_{3}^{M}\right)}}{b_{1}\left(t_{1}^{m}\right)}+\frac{\bar{p} S_{1}\left(t_{1}^{m}\right)}{b_{1}\left(t_{1}^{m}\right) e^{M_{1}}}-\bar{d},
$$

which implies

$$
\left[\frac{\bar{q}}{\bar{p}}+\frac{\bar{\beta}}{\bar{p}}+\frac{c\left(t_{1}^{m}\right)}{b_{1}\left(t_{1}^{m}\right)}\right] e^{u_{3}\left(t_{3}^{M}\right)} \geq \frac{a_{1}\left(t_{1}^{m}\right)-D_{1}\left(t_{1}^{m}\right)+S_{1}\left(t_{1}^{m}\right) e^{-M_{1}}}{b_{1}\left(t_{1}^{m}\right)}-\frac{\bar{d}}{\bar{p}} .
$$

Therefore,

$$
\left[K+\left(\frac{c}{b_{1}}\right)^{u}\right] e^{u_{3}\left(t_{3}^{M}\right)} \geq K^{*}-\frac{\bar{d}}{\bar{p}}
$$

That is,

$$
u_{3}\left(t_{3}^{M}\right) \geq \ln \frac{K^{*}-\bar{d} / \bar{p}}{K+\left(c / b_{1}\right)^{u}}:=m_{5}^{*} .
$$


It follows from(2.48) and (2.66)that

$$
u_{3}\left(t_{3}^{m}\right) \geq u_{3}\left(t_{3}^{M}\right)-\int_{0}^{T}\left|u_{3}^{\prime}(t)\right| d t \geq m_{5}^{*}-2 T\left(\bar{d}+\bar{q} \widetilde{M}_{0}+\bar{\beta} \widetilde{M}_{0}\right) .
$$

It follows from (2.55), (2.60), and (2.67) that Claim 4 holds.

Clearly, one of the following inequalities holds:

(i) $M_{1}^{*}>m_{2}^{*}$,

(ii) $M_{1}^{*} \leq m_{2}^{*}$.

Since $m_{1}^{*}<M_{1}^{*}$ and $m_{2}^{*} \leq M_{2}^{*}$, (ii) implies $M_{2}^{*}>m_{1}^{*}$. Thus, according to Claims $1-3$, one of the following four cases must hold:

$\left(\mathrm{P}_{1}\right) m_{1} \leq m_{1}^{*}-2 T\left(\bar{D}_{1}+\bar{b}_{1} M_{0}+\bar{c} \widetilde{M}_{0}\right) \leq u_{1}\left(t_{1}^{m}\right) \leq u_{2}\left(t_{2}^{m}\right), u_{2}\left(t_{2}^{M}\right) \leq u_{1}\left(t_{1}^{M}\right) \leq M_{1}^{*} \leq M_{1} ;$

$\left(\mathrm{P}_{2}\right) m_{1} \leq m_{2}^{*} \leq u_{2}\left(t_{2}^{m}\right)<u_{1}\left(t_{1}^{m}\right), u_{2}\left(t_{2}^{M}\right) \leq u_{1}\left(t_{1}^{M}\right) \leq M_{1}^{*} \leq M_{1}$;

$\left(\mathrm{P}_{3}\right) m_{1} \leq m_{1}^{*}-2 T\left(\bar{D}_{1}+\bar{b}_{1} M_{0}+\bar{c} \widetilde{M}_{0}\right) \leq u_{1}\left(t_{1}^{m}\right) \leq u_{2}\left(t_{2}^{m}\right), u_{1}\left(t_{1}^{M}\right)<u_{2}\left(t_{2}^{M}\right) \leq M_{2}^{*} \leq$ $M_{1}$;

$\left(\mathrm{P}_{4}\right) m_{1} \leq m_{2}^{*} \leq u_{2}\left(t_{2}^{m}\right)<u_{1}\left(t_{1}^{m}\right), u_{1}\left(t_{1}^{M}\right)<u_{2}\left(t_{2}^{M}\right) \leq M_{2}^{*} \leq M_{1}$.

From this and Claims 3 and 4 , we have

$$
\max _{t \in[0, T]}\left|u_{i}(t)\right| \leq \max \left\{\left|M_{1}\right|,\left|M_{2}\right|,\left|m_{1}\right|,\left|m_{2}\right|\right\}:=M^{*}, \quad i=1,2,3
$$

Obviously, $M^{*}$ is independent of $\lambda$.

Set

$$
B_{i}^{*}:=\bar{a}_{i}+\sqrt{\left(\bar{a}_{i}\right)^{2}+4 \bar{b}_{i} \bar{S}_{i}}, \quad i=1,2
$$

Take sufficiently large $M$ such that

$$
\begin{gathered}
M>3 \max \left\{M^{*},\left|m_{1}^{*}\right|,\left|m_{2}^{*}\right|,\left|m_{3}^{*}\right|,\left|m_{4}^{*}\right|,\left|m_{5}^{*}\right|\right\}, \\
M>\left|v_{1}^{*}\right|+\left|v_{2}^{*}\right|+\left|v_{3}^{*}\right|,
\end{gathered}
$$

where

$$
\begin{gathered}
v_{1}^{*}=\ln \frac{B_{1}^{*}}{2 \bar{b}_{1}}, \quad v_{2}^{*}=\ln \frac{B_{2}^{*}}{2 \bar{b}_{2}}, \\
v_{3}^{*}=\ln \frac{\bar{p} B_{1}^{*}+\sqrt{\left[\bar{p} B_{1}^{*}\right]^{2}+16\left(\bar{b}_{1}\right)^{2}[\bar{q}+\bar{\beta}] \bar{S}_{3}}}{4 \bar{b}_{1}[\bar{q}+\bar{\beta}]} .
\end{gathered}
$$

Clearly, the condition (i) in Lemma 2.1 is satisfied by system (2.7).

Define $H\left(u_{1}, u_{2}, u_{3}, \mu\right): \mathbb{R}^{3} \times[0,1] \rightarrow \mathbb{R}^{3}$ by

$$
H\left(u_{1}, u_{2}, u_{3}, \mu\right)=\left(\begin{array}{c}
\bar{a}_{1}-\bar{b}_{1} e^{u_{1}}+\frac{\bar{S}_{1}}{e_{u_{1}}^{u_{1}}} \\
\bar{a}_{2}-\bar{b}_{2} e^{u_{2}}+\frac{\bar{S}_{2}}{e^{u_{2}}} \\
\bar{p} e^{u_{1}}-[\bar{q}+\bar{\beta}] e^{u_{3}}+\frac{\bar{S}_{3}}{e^{u_{3}}}
\end{array}\right)+\mu\left(\begin{array}{c}
\bar{D}_{1} e^{u_{2}-u_{1}}-\bar{c} e^{u_{3}}-\bar{D}_{1} \\
\bar{D}_{2} e^{u_{1}-u_{2}}-\bar{D}_{2} \\
-\bar{d}
\end{array}\right) .
$$


We show that

$$
H\left(u_{1}, u_{2}, u_{3}, \mu\right) \neq 0 \quad \text { for any } u=\left(u_{1}, u_{2}, u_{3}\right) \in \partial B_{M}\left(\mathbb{R}^{3}\right), \mu \in[0,1] .
$$

Indeed, assume to the contrary, that

$$
H\left(u_{1}^{*}, u_{2}^{*}, u_{3}^{*}, \mu^{*}\right)=0 \quad \text { for some } u^{*}=\left(u_{1}^{*}, u_{2}^{*}, u_{3}^{*}\right) \in \partial B_{M}\left(\mathbb{R}^{3}\right), \mu^{*} \in[0,1]
$$

Then, there exist $t_{i} \in[0, T], i=1,2$, such that

$$
\begin{gathered}
a_{1}\left(t_{1}\right)-b_{1}\left(t_{1}\right) e^{u_{1}^{*}}+\frac{S_{1}\left(t_{1}\right)}{e^{u_{1}^{*}}}+\mu^{*} D_{1}\left(t_{1}\right) e^{u_{2}^{*}-u_{1}^{*}}-\mu^{*} c\left(t_{1}\right) e^{u_{3}^{*}}-\mu^{*} D_{1}\left(t_{1}\right)=0, \\
a_{2}\left(t_{2}\right)-b_{2}\left(t_{2}\right) e^{u_{2}^{*}}+\frac{S_{2}\left(t_{2}\right)}{e^{u_{2}^{*}}}+\mu^{*} D_{2}\left(t_{2}\right) e^{u_{1}^{*}-u_{2}^{*}}-\mu^{*} D_{2}\left(t_{2}\right)=0, \\
-\mu^{*} \bar{d}+\bar{p} e^{u_{1}^{*}}-[\bar{q}+\bar{\beta}] e^{u_{3}^{*}}+\frac{\bar{S}_{3}}{e^{u_{3}^{*}}}=0 .
\end{gathered}
$$

By using the arguments of (2.19), (2.20), (2.27), (2.38), (2.44), (2.54), (2.59), (2.66), one can prove that

$$
\left|u_{i}^{*}\right| \leq \max \left|M_{1}\right|,\left|M_{2}\right|,\left|m_{1}^{*}\right|,\left|m_{2}^{*}\right|,\left|m_{3}^{*}\right|,\left|m_{4}^{*}\right|,\left|m_{5}^{*}\right|, \quad i=1,2,3,
$$

which implies that $\left\|u^{*}\right\|=\left|u_{1}^{*}\right|+\left|u_{2}^{*}\right|+\left|u_{3}^{*}\right| \leq 3 \max \left\{M^{*},\left|m_{1}^{*}\right|,\left|m_{2}^{*}\right|,\left|m_{3}^{*}\right|,\left|m_{4}^{*}\right|\right.$, $\left.\left|m_{5}^{*}\right|\right\}<M$. This contradicts the fact that $u^{*} \in \partial B_{M}\left(\mathbb{R}^{3}\right)$. Therefore, $H\left(u_{1}, u_{2}, u_{3}, \mu\right)$ is a homotopy.

Since

$$
g(u)=\left(\begin{array}{c}
\bar{a}_{1}-\bar{D}_{1}-\bar{b}_{1} e^{u_{1}}-\bar{c} e^{u_{3}}+\bar{D}_{1} e^{u_{2}-u_{1}}+\frac{\bar{S}_{1}}{e^{u_{1}}} \\
\bar{a}_{2}-\bar{D}_{2}-\bar{b}_{2} e^{u_{2}}+\bar{D}_{2} e^{u_{1}-u_{2}}+\frac{\bar{S}_{2}}{e^{u_{2}}} \\
-\bar{d}+\bar{p} e^{u_{1}}-[\bar{q}+\bar{\beta}] e^{u_{3}}+\frac{\bar{S}_{3}}{e^{u_{3}}}
\end{array}\right)=H\left(u_{1}, u_{2}, u_{3}, 1\right)
$$

$g(u) \neq 0$ for any $\left(u_{1}, u_{2}, u_{3}\right) \in \partial B_{M}\left(\mathbb{R}^{3}\right)$. Thus, the condition (ii) in Lemma 2.1 is satisfied. Next we show that condition (iii) also holds. It is easy to see that $H\left(u_{1}, u_{2}, u_{3}, 0\right)=0$ has a unique solution $v^{*}=\left(v_{1}^{*}, v_{2}^{*}, v_{3}^{*}\right)$, where $v_{1}^{*}, v_{2}^{*}, v_{3}^{*}$ are the same as those in (2.71). Clearly, $\left\|v^{*}\right\|=\left|v_{1}^{*}\right|+\left|v_{2}^{*}\right|+\left|v_{3}^{*}\right|<M$, that is, $v^{*} \in B_{M}\left(\mathbb{R}^{3}\right)$. According to the invariance of homotopy, we obtain

$$
\operatorname{deg}\left(g, B_{M}\left(\mathbb{R}^{3}\right)\right)=\operatorname{deg}\left(H(\cdot, 1), B_{M}\left(\mathbb{R}^{3}\right)\right)=\operatorname{deg}\left(H(\cdot, 0), B_{M}\left(\mathbb{R}^{3}\right)\right)=-1 .
$$

Therefore, all of the conditions required in Lemma 2.1 hold. According to Lemma 2.1, system (2.7) has one $T$-periodic solution $\left(u_{1}^{*}(t), u_{2}^{*}(t), u_{3}^{*}(t)\right)^{T}$. It is easy to see that $\left(x_{1}^{*}(t)\right.$, $\left.x_{2}^{*}(t), y^{*}(t)\right)^{T}=\left(\exp \left[\left(u_{1}^{*}(t)\right], \exp \left[u_{2}^{*}(t)\right], \exp \left[u_{3}^{*}(t)\right]\right)^{T}\right.$ is a positive $T$-periodic solution of system (1.1). By the arguments similar to Claims 1-4, one can show

$$
m_{1} \leq u_{i}^{*}(t) \leq M_{1} \quad(i=1,2), \quad m_{2} \leq u_{3}^{*}(t) \leq M_{2}, \quad t \geq 0,
$$


which implies

$$
m_{0} \leq x_{i}^{*}(t) \leq M_{0} \quad(i=1,2), \quad \tilde{m}_{0} \leq y^{*}(t) \leq \widetilde{M}_{0}, \quad t \geq 0 .
$$

The proof is complete.

Consider the special case of system (1.1) that $S_{i}(t) \equiv 0, i=1,2,3$. In this case, by Theorem 2.2, we have the following.

Corollary 2.3. In addition to $\left(\mathrm{H}_{1}\right)$ and $\left(\mathrm{H}_{2}\right)$, assume further that system (1.1) satisfies one of the following conditions:

$\left(\mathrm{H}_{3}\right)^{\prime}\left(a_{i} / b_{i}\right)^{l}>\bar{d} / \bar{p}, i=1,2$;

$\left(\mathrm{H}_{4}\right)^{\prime}\left(\left(a_{1}-D_{1}\right) / b_{1}\right)^{l}>\bar{d} / \bar{p}$.

Then system (1.1) has at least one positive T-periodic solution.

Remark 2.4. Corollary 2.3 greatly improves [15, Theorem 2.1] and [5, Theorem 1.1].

\section{Acknowledgment}

This work was supported by the National Natural Science Foundation of China (no. 10161007; 10561004; 10271044).

\section{References}

[1] E. Beretta and Y. Kuang, Convergence results in a well-known delayed predator-prey system, Journal of Mathematical Analysis and Applications 204 (1996), no. 3, 840-853.

[2] F. Brauer and A. C. Soudack, Coexistence properties of some predator-prey systems under constant rate harvesting and stocking, Journal of Mathematical Biology 12 (1981), no. 1, 101-114.

[3] _ On constant effort harvesting and stocking in a class of predator-prey systems, Journal of Theoretical Biology 95 (1982), no. 2, 247-252.

[4] J. Cao, Q. Li, and S. Wan, Periodic solutions of the higher-dimensional non-autonomous systems, Applied Mathematics and Computation 130 (2002), no. 2-3, 369-382.

[5] S. Chen, J. Zhang, and T. Young, Existence of positive periodic solution for nonautonomous predator-prey system with diffusion and time delay, Journal of Computational and Applied Mathematics 159 (2003), no. 2, 375-386.

[6] H. I. Freedman and J. H. Wu, Periodic solutions of single-species models with periodic delay, SIAM Journal on Mathematical Analysis 23 (1992), no. 3, 689-701.

[7] W. Krawcewicz and J. Wu, Theory of Degrees with Applications to Bifurcations and Differential Equations, Canadian Mathematical Society Series of Monographs and Advanced Texts, John Wiley \& Sons, New York, 1997.

[8] Y. Kuang, Delay Differential Equations with Applications in Population Dynamics, Mathematics in Science and Engineering, vol. 191, Academic Press, Massachusetts, 1993.

[9] A. Leung, Periodic solutions for a prey-predator differential delay equation, Journal of Differential Equations 26 (1977), no. 3, 391-403.

[10] Y. Li, Periodic solutions of a periodic delay predator-prey system, Proceedings of the American Mathematical Society 127 (1999), no. 5, 1331-1335.

[11] X. Song and L. Chen, Persistence and global stability for nonautonomous predator-prey system with diffusion and time delay, Computers \& Mathematics with Applications 35 (1998), no. 6, 33-40.

[12] Y. Xia and J. Cao, Almost periodicity in an ecological model with $M$-predators and $N$-preys by "pure-delay type" system, Nonlinear Dynamics 39 (2005), no. 3, 275-304. 


\section{Periodic solutions for predator-prey patch systems}

[13] Y. Xia, J. Cao, H. Zhang, and F. Chen, Almost periodic solutions of $n$-species competitive system with feedback controls, Journal of Mathematical Analysis and Applications 294 (2004), no. 2, 503-522.

[14] Z. Yang and J. Cao, Positive periodic solutions of neutral Lotka-Volterra system with periodic delays, Applied Mathematics and Computation 149 (2004), no. 3, 661-687.

[15] Z. Q. Zhang and Z. Wang, Periodic solutions for nonautonomous predator-prey system with diffusion and time delay, Hiroshima Mathematical Journal 31 (2001), no. 3, 371-381.

Hui Fang: Faculty of Information Science and Engineering, Ningbo Institute of Technology,

Zhejiang University, Ningbo, Zhejiang 315100, China

E-mail address: huifang@public.km.yn.cn

Zhicheng Wang: Department of Applied Mathematics, Hunan University, Changsha 410082, China E-mail address: zcwang2004@163.com 


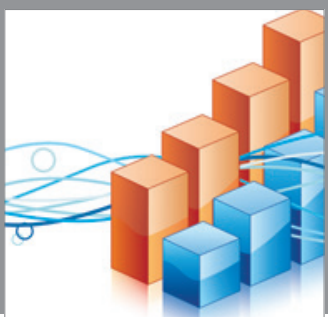

Advances in

Operations Research

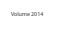

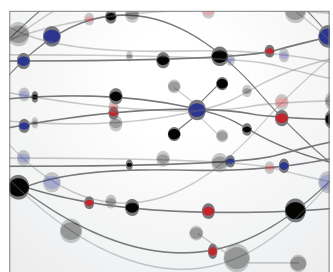

\section{The Scientific} World Journal
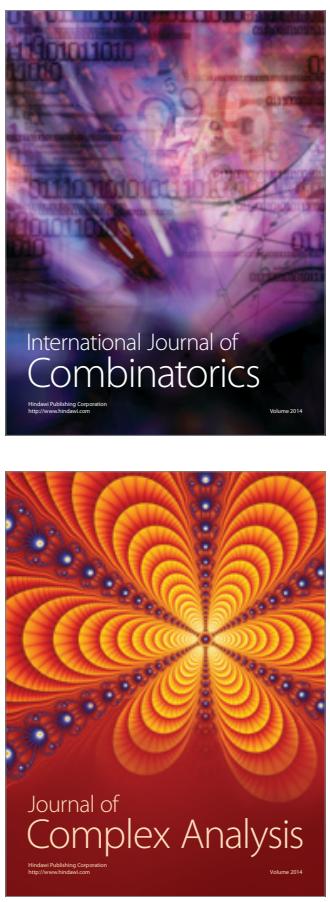

International Journal of

Mathematics and

Mathematical

Sciences
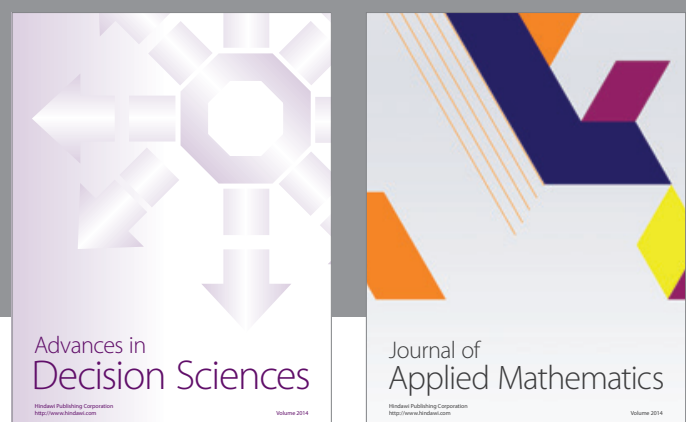

Journal of

Applied Mathematics
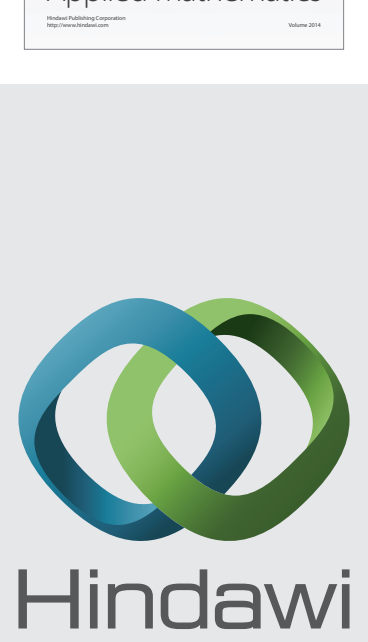

Submit your manuscripts at http://www.hindawi.com
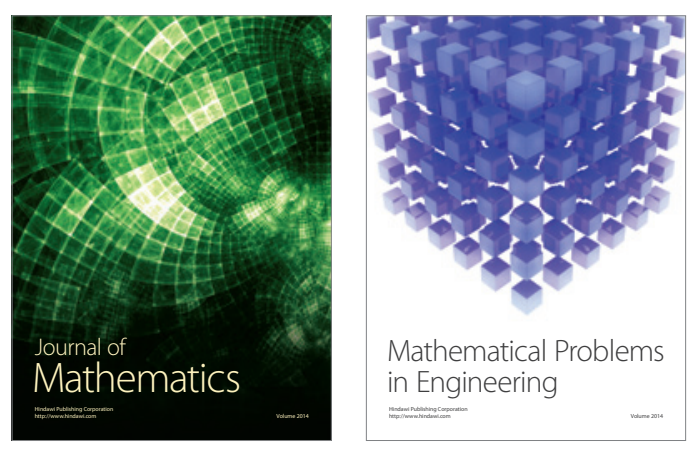

Mathematical Problems in Engineering
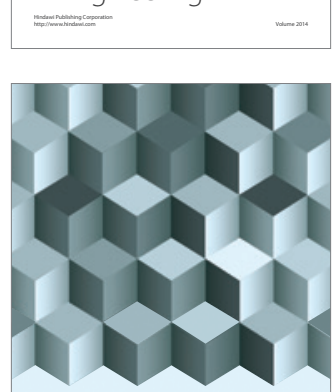

Journal of

Function Spaces
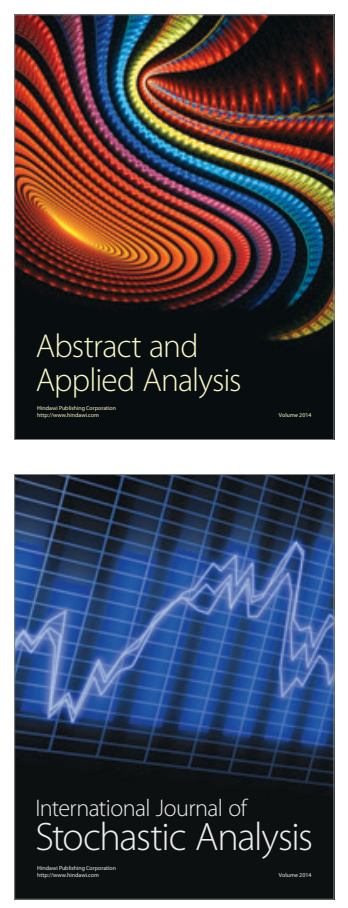

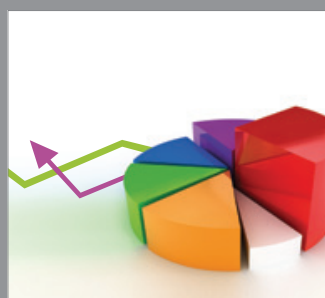

ournal of

Probability and Statistics

Promensencen
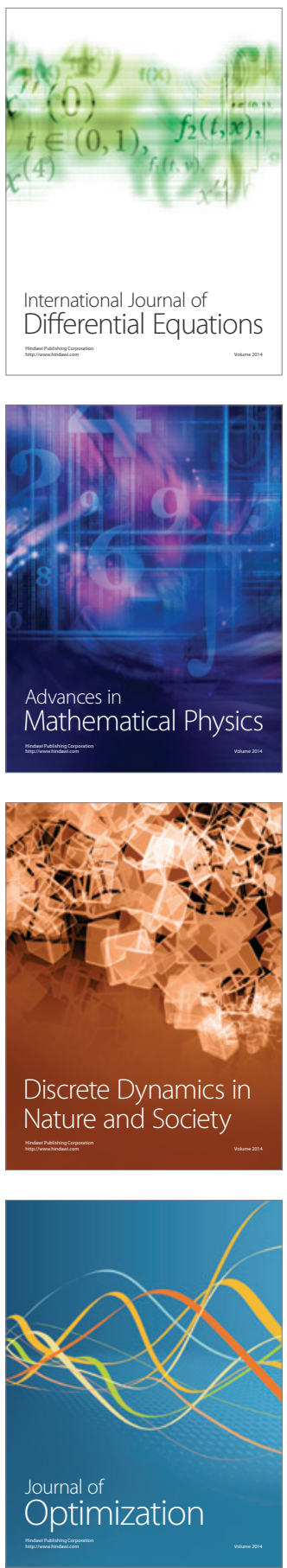